\title{
La distribución normal en ciencias biomédicas: un enfoque a partir de las distribuciones de Pearson
}

\section{The normal distribution in biomedical sciences: an approach from the Pearson's distributions}

\section{Distribución normal y distribuciones de Pearson}

\author{
Rafael M. Ávila Ávila. ${ }^{(1)}$ \\ Julio César Pino Tarragó. ${ }^{(2)}$ \\ María del Carmen Expósito Gallardo. (3) \\ Dunia Lisbet Domínguez Gálvez. ${ }^{(4)}$
}

(1) Universidad de Holguín. Cuba, ravilaa@ uho.uho.edu.cu

(2) Universidad Estatal del Sur de Manabí. Ecuador, jpinoecuador@gmail.com

(3) Universidad Médica Mariana Grajales Coello. Cuba, mariaex@infomed.sld.cu

(4) Universidad Estatal del Sur de Manabí. Ecuador, juliodunia1406@gmail.com

Contacto: ravilaa@uho.edu.cu

\section{Resumen}

La distribución normal es una de las más importantes en el proceso de enseñanza aprendizaje de la Bioestadística para la especialidad de Medicina. Sin embargo, la ausencia de un enfoque deductivo en la introducción al tópico, dificulta la comprensión de su origen. A partir de sistemas de distribuciones continuas de probabilidad, se propone una vía alternativa para presentarla, tanto en la forma general como en la estándar. Teniendo en cuenta los métodos analíticos sintéticos y la revisión documental, se identifican los vacío epistémicos y las dificultades en el tratamiento del tema. Los objetivos esenciales consisten en deducir la expresión matemática de tal distribución y valorar la posibilidad de introducir los elementos cualitativos básicos del procedimiento usado, en el programa de Informática Médica II, enfatizando en las nociones del Cálculo Diferencial, en etapa previa a los elementos del Cálculo Integral. La conclusión fundamental se asocia a la contribución del enfoque sugerido, al rigor y a la armonía al conocimiento estadístico matemático en la disciplina, si bien se precisa de conceptos del Análisis Diferencial, los cuales favorecen la comprensión de las aplicaciones prácticas tanto en el ámbito de la carrera de Medicina como en otras áreas de investigación de las ciencias biomédicas.

Palabras claves: Bioestadística, distribución normal, distribuciones de Pearson, enseñanza aprendizaje.

\section{Abstract}

The normal distribution is one of the most important in the teaching learning process of Biostatistics for the specialty of Medicine. However, the absence of a deductive approach in the introduction to the topic, makes difficult to understand its origin. From the starting point of the systems of continue distributions of probability, it is proposed an alternative way to introduce such distribution in general and standard form. Some epistemic gaps are identified in the treatment of the thematic, taking into account the analytic synthetic and documentary review methods. The essential objectives consist of the deduction the mathematical expression of such distribution as well as to value the possibility to introduce the basic elements of the used procedure, in the Program of the Medical Informatics II, emphasizing the notions of Differential Calculus in previous stage to the elements of Integral Calculus. The essential conclusion is associated to the contribution of the suggested approach to the rigor and harmony of mathematical statistic knowledge in the discipline, although some concepts of Differential Analysis are necessary which favour the understanding the practical applications in the career of Medicine and in other branches of the research in biomedical sciences.

Keywords: Biostatistics, normal distribution, Pearson's distributions, teaching learning.

\section{Introducción}

La distribución normal es tal vez la más importante en el ámbito de la Estadística clásica. Varias razones de este modelo teórico, denominado además segunda Ley de Laplace, distribución 
laplaciana, distribución de Gauss-Laplace o simplemente distribución de Gauss, ameritan su conocimiento detallado. El mismo es esencial no sólo en el contexto de la teoría de los errores de las mediciones. En el marco de la estadística inferencial y en el análisis del comportamiento de variables aleatorias en las ciencias médicas y biológicas, dichas variables en muchos casos se rigen muy bien por tal modelo (Torres at al., 2004).

El papel fundamental de la distribución gaussianolaplaceana reside en que bajo amplias suposiciones, al incrementarse el número de sumandos, resulta asintóticamente normal. Ello está vinculado al teorema del límite central. La importancia de este radica en ofrecer una explicación al hecho de que "si el resultado de un experimento aleatorio se determina con un gran número de factores aleatorios y la influencia de cada uno de ellos es tan pequeña que puede despreciarse, entonces tal experimento se aproxima con éxito mediante una distribución normal, siendo de manera adecuada la esperanza matemática y la varianza" (Koroliuk, 1986).

Existen varias formas de abordar el estudio de esta distribución, cuestión expuesta detalladamente en algunos libros clásicos acerca de la teoría de las probabilidades (Gnedenko, 1997; Whittle 1982). No obstante, desde una perspectiva histórica, fue introducida y usada sistemáticamente por Carl Friedrich Gauss (1777-1855) en relación con los fundamentos teóricos para el análisis de los errores de las mediciones físicas. Otros autores la definen simplemente sin que medie discusión sobre su origen (Guerra, Menéndez, Barrera, Egaña, 1991).

En otros textos (Freund, 2007) se presenta como curva de distribución continua que da una aproximación a la distribución binomial en el caso en que el número de ensayos $\mathrm{n}$ sea grande y la probabilidad de éxito sea cercana a un medio (1/2). También se introduce a partir de la definición de espacio probabilístico absolutamente continuo de modo que cualquier función no negativa, cuya integral por todo el eje real, o sea, desde menos infinito $(-\infty)$ hasta más infinito $(-\infty)$, sea igual 1 , constituye la densidad de distribución de cierta variable aleatoria (Chistiakov, 1982).

Una interesante familiarización sobre su empleo en Bioestadística y Medicina se logra a partir de las nociones de Cálculo Integral y razonamientos geométricos. En tal caso, la función de densidad aparece como forma de expresión de las distribuciones de frecuencias teóricas apelando a la idea de dependencia funcional con respecto a cierta variable aleatoria x (Torres at al., 2004). Luego la presentación de la expresión matemática en función tanto de $\mathrm{x}$, como de los parámetros poblacionales media $(\mu)$ y desviación estándar $(\sigma)$, se introduce como la síntesis del conocimiento imprescindible para la comprensión ulterior de los tópicos estadísticos, si bien se detectan vacíos epistémicos.

\section{Distribución normal y noción de integración: algunas dificultades para su comprensión}

El concepto de integral, uno de los más importantes en el Análisis Matemático. En particular, el de integral definida así como su interpretación, favorecen la comprensión de ideas asociadas al empleo de la Estadística en las ciencias biomédicas. Sin embargo, los estudiantes de Medicina en el nivel de pregrado, exhiben un pobre dominio sobre dicho concepto. Por otra parte, tienen una incipiente formación con respecto a las nociones de función, límite, continuidad, cálculo diferencial, cálculo integral en general así como de la relación entre las operaciones de derivación e integración de funciones.

La noción de integral definida, su interpretación geométrica en términos de área bajo la curva y su relación directa con la probabilidad no resulta evidente. El esfuerzo se centra en afirmar que el aumento indefinido del número de observaciones implica la aproximación del histograma y el polígono de frecuencia a la curva ideal o modelo teórico de distribución de probabilidad. Aparece así de manera subrepticia, la noción de límite, que no se aprovecha en todas su potencialidades.

No obstante, se reafirma el hecho de que la aproximación referida implica que el área de cada rectángulo se acerque a la frecuencia teórica o "probabilidad de que el valor de una observación de la variable se encuentre en dicho intervalo"(Torres at al., 2004). Ello conduce a la interpretación correspondiente: el área bajo la curva en cierto intervalo $(a, b)$ como probabilidad de que la variable aleatoria tome valores entre a y b. Así el área total no es más que la probabilidad de que tal variable adopte siempre un valor entre todo el conjunto de los observados y el mimo sea la unidad.

En el razonamiento anterior está implícito el concepto de función $\mathrm{f}(\mathrm{x})$ como medio de expresión de las distribuciones teóricas. Estas se vinculan con la función $\mathrm{f}(\mathrm{x})$ cuya integral definida entre los valores $\mathrm{x}=\mathrm{a} \mathrm{y} \mathrm{x}=\mathrm{b}$ coincide con el áreas limitada por el gráfico de dicha función $\mathrm{f}(\mathrm{x})$, $\mathrm{y}$ las rectas $\mathrm{x}=\mathrm{a}$ y $\mathrm{x}=\mathrm{b}$. La expresión simbólica de tales hechos es dada en la forma: 


$$
P(a<x<b)=\int_{a}^{b} f(x) d x
$$

Y de igual manera, si el valor de la probabilidad es uno, entonces dicha probabilidad coincide con la del evento seguro. Esto queda expresado por la condición de normalización:

$$
\int_{\infty}^{\infty} f(x) d x=1
$$

Muy a pesar del valioso y considerable esfuerzo para propiciar la comprensión de tales conocimientos, una especie de ruptura tiene lugar cuando se presenta la expresión para el cálculo de probabilidades. Y ello es debido que la función de densidad correspondiente a la distribución normal, se presente únicamente formando parte de la siguiente fórmula, que es conocida por matemáticos o por aquellos que tienen nociones algo más avanzadas y no para los futuros galenos:

$$
P(a<x<b)=\int_{a}^{b} \frac{1}{\sigma \sqrt{2 \pi}} e^{-\frac{1}{2}\left(\frac{x-\mu}{\sigma}\right)^{2}} d x
$$

El énfasis ahora se traslada al conocimiento de los parámetros poblacionales $\sigma \mathrm{y} \mu$, en aras de eludir el cálculo de la integral. Esta problemática queda aparentemente resuelta, al sugerirse tablas para determinar las probabilidades de que cierto valor de $\mathrm{x}$ se encuentre contenido entre los límites a y $\mathrm{b}$. Por otra parte, se sugiere el empleo de otras propiedades de la integral, que resultan algo esotéricas en un primer conocimiento del tema para futuros profesionales de la Medicina..

Es menos arduo, inferir la forma matemática de la distribución normal estándar para el caso de que $\mu=0$ y $\sigma=1$. La simple sustitución, no debe dificulta la obtención de la siguiente función de densidad:

$$
f(x)=\frac{1}{\sqrt{2 \pi}} e^{-\frac{x^{2}}{2}}, \quad-\infty<x<+\infty
$$

En general, el enfoque citado no hace referencia a procedimiento alguno que posibilite deducir la forma matemática concreta de la función de densidad. Esto representa un vacío epistémico que rompe la coherencia deductiva que caracteriza la exposición del material docente. En consecuencia, el conocimiento matemático mínimo asociado a la Estadística se presenta a los estudiantes de Medicina de manera incompleta.

Una vía para el tratamiento conjunto de distribuciones de probabilidad continuas consiste en el empleo de sistemas de distribuciones. Las mismas verifican ecuaciones diferenciales de manera que las distribuciones concretas se obtienen por vía deductiva. Así se logra aplicar sistemáticamente metodologías para la generación de familias de distribuciones y obtener características comunes a dichas familias (Herrerías y Callejón, 2017).

El principal objetivo del artículo consiste en deducir la función de densidad de la distribución normal, a partir de uno de los sistemas de distribuciones continuas univariantes más estudiados, introducido por Karl Pearson (18571936); junto a este, el otro propósito es valorar la posibilidad de introducir dicha función acudiendo a las nociones del cálculo diferencial, antes de exponer las asociadas a la integral definida, de modo que pueda resultar de utilidad en la enseñanza de la Bioestadística y en el quehacer didáctico de los docentes que imparten la asignatura. De igual forma, formular algunas recomendaciones asociadas a la enseñanza de las aplicaciones de la distribución normal a los estudiantes de Medicina, resultó un objetivo de interés.

\section{Materiales y métodos}

Los métodos empleados corresponden al nivel teórico. En este caso, el rol crucial lo desempeña el método analítico sintético, identificado en ocasiones con el análisis y la síntesis.

En un ámbito más general, la metodología consideró como materiales imprescindibles, los obtenidos a partir de la recopilación documental (Ander-Eg, 1995; Giacosa et al., 2015). Ello implicó una exhaustiva revisión como punto de partida para el procesamiento de la información asociada a: planes de estudios; diferentes normativas del Ministerio de Educación (MES); programas analíticos de las diferentes disciplinas y asignaturas que integran dichos planes para la especialidad de Medicina y su bibliografía recomendada y en el contexto de las experiencias acumuladas por el autor principal durante el proceso de enseñanza aprendizaje de los contenidos en la Universidad Médica de Holguín. De igual manera, otros libros sobre Estadística 
Matemática que se referenciaron en el apartado introductorio, resultaron de gran utilidad

Los métodos propios de la Matemática, como el deductivo, fueron provechosos para la obtención de la forma explícita de la función de densidad de distribución normal adoptando como punto de partida las distribuciones de Pearson. El procedimiento para determinar el tipo de distribución dentro de los doce tipos que pertenecen a esta categoría y que aproxima los datos observados, requirió del cálculo de los cuatro primeros momentos y luego las estimaciones de los parámetros correspondientes.

\section{Resultados}

Deducción de la función de densidad de distribución normal a partir de las distribuciones de Pearson

La familia de distribuciones de Pearson, introducida por este matemático entre 1894 y 1895 , proporciona una descripción flexible de distintas distribuciones. Estas se identifican comúnmente en investigaciones biométricas. El propio Pearson introdujo nuevas clases en 1916.

La ecuación diferencial que definen las distribuciones de Pearson (Koroliuk,1986), tiene el siguiente aspecto:

$$
\frac{d f(x)}{d x}=\frac{a_{1} x+a_{0}}{b_{0}+2 b_{1} x+b_{2} x^{2}} f(x)
$$

En la expresión anterior: $\mathrm{a}_{0}, \mathrm{a}_{1}, \mathrm{~b}_{0}, \mathrm{~b}_{1}, \mathrm{~b}_{2}$ constituyen los parámetros de la distribución que están determinados completamente con los cuatro primeros momentos centrales.

La aproximación más general a los momentos, parte del concepto de esperanza matemática $\mathrm{E}(\mathrm{x}) \mathrm{o}$ media probabilística. Si la variable aleatoria $X$ es discreta $\mathrm{y}$ tiene valores $\mathrm{X}_{1}, \mathrm{X}_{2}, \ldots \ldots, \mathrm{X}_{\mathrm{k}}$ con probabilidad $\mathrm{P}_{1}, \mathrm{P}_{2}, \ldots ., \mathrm{P}_{\mathrm{k}}$, en este caso, dicha esperanza se define como la suma de los productos de los valores que toma la variable por sus probabilidades correspondientes, es decir:

$$
E X=p_{1} x_{1}+p_{2} x_{2}+p_{3} x_{3}+\ldots . .+p_{n} x_{n}=\sum_{k=1}^{n} p_{k} x_{k}
$$

En el caso de que la variable aleatoria sea continua con densidad de probabilidad $\mathrm{f}(\mathrm{x})$, entonces la suma se sustituye por una integral de manera que la expresión resulta:

$$
E X=\int_{-\infty}^{+\infty} x f(x) d x
$$

Los momentos k-ésimos de la magnitud $\mathrm{X}$ y el késimo momento central de la magnitud X-EX (si existe la esperanza matemática), se pueden expresar también a través de integrales cuando las variables aleatorias son continuas.

El cálculo de tales integrales, permite concluir que: el primer momento $(\mathrm{k}=1)$ de $\mathrm{X}$ resulta precisamente la esperanza matemática $\left(\mathrm{MX}^{1}=\mathrm{EX}\right)$, mientras que en segundo momento central $(k=2)$ de $\mathrm{X}-\mathrm{MX}\left(\mathrm{M}(\mathrm{X}-\mathrm{MX})^{2}=\mathrm{DX}\right)$ es la varianza.

La magnitud $\sigma=\sqrt{D X} \quad$ constituye precisamente la desviación estándar de la magnitud $X$. Si la varianza se anula (DX=0), la probabilidad de que la variable aleatoria $X$ tome el valor igual a su esperanza matemática $(\mathrm{P}(\mathrm{x}=\mathrm{EX}))$ es igual a la unidad, anulándose en consecuencia la desviación estándar. Este hecho representa una de sus propiedades más importantes. El cálculo de MX y DX para el caso específico de la distribución normal corrobora tales resultados.

Por tanto, la esperanza matemática para una variable aleatoria que se distribuye normalmente es la media o promedio $\mu$, mientras que su varianza es $\sigma^{2}$. Tales parámetros se refieren al nivel poblacional. Si una magnitud aleatoria se describe por una distribución de tipo Pearson que satisface la ecuación (5), los coeficientes $a_{0}, b_{0}, b_{1}, b_{2}$, están relacionados con los momentos $\mathrm{M}_{\mathrm{k}} \mathrm{y} \mathbf{M}^{\mathrm{k}}$ de dicha distribución. Así $\mathrm{a}_{0}, \mathrm{~b}_{0}$ se relacionan mediante las siguientes expresiones:

$$
\begin{array}{r}
a_{0}=\frac{M_{3}\left(M_{4}+3 M_{2}^{2}\right)}{P_{M_{2} M_{3} M_{4}}} \\
b_{0}=-\frac{M_{2}\left(4 M_{2} M_{4}-3 M_{3}^{2}\right)}{P_{M_{2} M_{3} M_{4}}}
\end{array}
$$

$\mathrm{P}_{\text {M2M3M4 }}$ : es un polinomio en $\mathrm{M}_{2}, \mathrm{M}_{3}$ y $\mathrm{M}_{4}$ tal que:

$$
P_{M_{2} M_{3} M_{4}}=10 M_{4} M_{2}-18 M_{2}^{3}-12 M_{3}^{2}
$$

Si $a_{1}=1$ la ecuación (5) resulta 


$$
\frac{d f(x)}{d x}=\frac{x+a_{0}}{b_{0}+2 b_{1} x+b_{2} x^{2}} f(x)
$$

Si además $b_{1}=0, \quad b_{2}=0$, resolviendo la ecuación diferencial resultante, se obtiene:

$$
f(x)=C e^{\frac{\left(x+a_{0}\right)^{2}}{2 b_{0}}}
$$

$\mathrm{C}$ es una constante. Si se tiene en cuenta que $\mathrm{M}_{1}=0$, $\mathrm{M}_{2}=\sigma^{2}, \mathrm{M}_{3}=0, \mathrm{M}_{4}=3 \sigma^{4}$, sustituyendo en las expresiones (8) se obtiene que: $\mathrm{a}_{0}=0$ y $\mathrm{b}_{0}=-\sigma^{2}$. Por tanto la función de densidad de probabilidad (11) adopta, después de aplicar la condición de normalización (2), una forma similar a la presentada en la literatura que sirve como libro de texto de Bioestadística y es la siguiente:

$$
f(x)=\frac{1}{\sigma \sqrt{2 \pi}} e^{-\frac{x^{2}}{2 \sigma^{2}}},-\infty<x<+\infty
$$

Si el parámetro $\sigma$ es unitario $(\sigma=1)$ se obtiene la distribución normal estándar. Varias distribuciones continuas satisfacen la ecuación diferencial (10) y se sabe que el conocimiento de los cuatro primeros momentos posibilita estimar los cuatro parámetros que aparecen en la misma, de manera que se dispone de una densidad, pero las estimaciones en general no son eficientes por realizarse mediante el método de los momentos (Herrerías y Callejón, 2017).

La ecuación diferencial (10) también se presenta en la literatura (Herrerías y Callejón, 2017; Weisstein, 2017) en la forma:

$$
\frac{d f(x)}{d x}=\frac{d-x}{b_{0}+2 b_{1} x+b_{2} x^{2}} f(x)
$$

El problema de encontrar $\mathrm{f}(\mathrm{x})$ nuevamente se reduce a integrar la ecuación anterior. En el caso en que $b_{2}=b_{1}=0$ la densidad de probabilidad es de tipo gaussiano y corresponde a una distribución normal. La sustitución en la expresión (13) conduce a la siguiente ecuación simplificada:

$$
\frac{d f(x)}{d x}=\frac{d-x}{b_{0}} f(x)
$$

La integración de (14) y la aplicación de la condición de normalización (2) ofrecen como resultado la forma más familiar para la densidad de probabilidad de la distribución normal. Esta queda expresada mediante la fórmula:

$$
f(x)=\frac{1}{\sqrt{2 \pi b_{o}}} e^{-\frac{(x-d)^{2}}{2 b_{0}}}
$$

\section{Discusión}

Tal y como se ha expuesto, las distribuciones de Pearson presuponen la noción de diferenciación pues se definen a partir de una ecuación diferencial que debe satisfacer la densidad de probabilidad o función de densidad $\mathrm{f}(\mathrm{x})$.

En la exposición de las Matemáticas Superiores, el Cálculo Diferencial antecede al Cálculo Integral, incluso una introducción a ellos (Valdés y Sánchez, 2011) o la exposición de sus simples nociones. La presentación de la función de distribución mediante esta vía, ofrece la alternativa de seguir el orden lógico clásico en la introducción de los conceptos asociados así como su concepción histórico-lógica.

La explicación del procedimiento de obtención de la forma concreta de la distribución teórica, necesita de las nociones vinculadas con el concepto de ecuación diferencial ordinaria de primer orden y sus métodos de solución, sobre lo cual existe abundante literatura, por ejemplo (Elgoltz, 2010; Dawkins, 2007). Estos métodos ni se contemplan en los programas ni constituyen una prioridad, mas su referencia ofrece la posibilidad de una discusión de tipo cualitativo acerca de la ligazón existente entre las dos operaciones mutuamente inversas del Cálculo: la derivación y la integración.

La precedencia de los fundamentos del Cálculo Diferencial, prepara el camino para la comprensión del aparato conceptual básico de la integración. Hay autores que han sugerido el tratamiento de algunos contenidos sin acudir a las derivadas (Escalona y Velázquez, 2012), sobre todo vinculados con problemas de optimización y que generan conocimientos con relación al Cálculo Diferencial. No obstante, en la alternativa que se analiza, se introduce el concepto de derivada como parte de una ecuación y ello pudiera complementar las ideas que sugieren dichos autores acerca de la resolución de problemas que contemplan funciones variables en el tiempo y su impacto en la formación del médico general, muchos (Escalona, 2013). 
En el enfoque expuesto, el cálculo de los coeficientes de la distribución se ha asociado a los momentos de las mismas mediante las expresiones (8) y (9). El concepto de momento se trata con anterioridad en los tópicos inherentes a la Estadística Descriptiva y aparece esbozado en la literatura básica de Informática Médica (Torres et al., 2004). Aquí se centra el análisis en las medidas que posibilitan conocer la forma de la distribución de los datos y con ello obtener una información más completa.

Es así que se hace referencia así a los momentos de orden $\mathrm{R}$ con respecto a cero y con respecto a la media denotados por $m_{R}$ y $M_{R}$ respectivamente. Estos promedios de las potencias de orden $\mathrm{R}$ de los datos directos o sus desviaciones son tales que para $\mathrm{R}=1$, el momento de orden 1 respecto a cero es simplemente $\mathrm{m}_{1} \mathrm{o}$ la media aritmética usual mientras $\mathrm{M}_{1}$ es nulo; sin embargo si $\mathrm{R}=2$ entonces $\mathrm{M}_{2}=\mathrm{S}^{2}$ que no es más que la varianza de los datos. En consecuencia, el programa prepara al estudiante con una información mínima sobre su significado.

La posibilidad de asociar estos parámetros a la familia de las distribuciones de Pearson y de al menos, mostrar su incorporación a las ecuaciones que dichas distribuciones satisfacen, pone de manifiesto el carácter coherente del conocimiento matemático y no su reducción a un marco contextualizado.

Las ecuaciones diferenciales (10) y (14) son equivalentes, con la salvedad de que los coeficientes se denotan de manera distinta y ello no altera la solución obtenida para la función de densidad.

\section{Algunas consideraciones sobre la enseñanza de la distribución normal en la carrera de Medicina}

Las variaciones al azar en poblaciones o muestras muy grandes suelen corresponder a ciertos tipos de distribuciones. Es recomendable mostrar las ideas básicas vinculadas a la deducción de su forma matemática.

La distribución normal se representa gráficamente por una curva a veces denominada curva de errores normales (Bowman y Raud, 1984). Se recomienda tener presente que los términos error y normal tienen sentidos muy especiales, de modo que no han de confundirse con los sentidos usuales atribuidos a estas palabras. Por tal motivo para hacer referencia a la curva o a la distribución, se sugiere emplear el vocablo gaussiana y discutir el sentido preciso de tales términos.
Entre las aplicaciones de la distribución normal durante en la carrera de Medicina, se identifica la vinculada a la definición de los rangos de normalidad de las variables aleatorias. Hay que tener presente que se trata de encontrar ciertos valores tales que multiplicados por la desviación estándar y luego, el resultado de tal multiplicación sumado y restado al promedio, permita obtener los rangos referidos, si se conocen los parámetros poblacionales y la proporción de enfermos en dicha población.

No obstante, la respuesta a situaciones problémicas asociadas a los rangos de normalidad, puede obtenerse de manera general a partir del planteamiento de un interesante problema matemático cuya solución requiere el conocimiento de los métodos de integración que están vedados en los programas de la asignatura Bioestadística. Estos, además, incluyen funciones que no se integran por vía elemental. Sin embargo en ciertos casos simplificados, se puede sugerir una vía menos laboriosa y ardua que el docente debe previamente seleccionar con sumo cuidado.

Se recomienda prestar especial atención a la aplicación de la estadística a variables aleatorias que siguen un comportamiento gaussiano y en situaciones en que las muestras son pequeñas. Ello ha de estar fundamentado rigurosamente para admitir que tal muestra ha sido seleccionada al azar de una población que se distribuye normalmente, pues de otra manera, los resultados inferidos pueden no ser válidos.

En caso en que se detecten desviaciones de una muestra grande respecto al comportamiento normal, es sugerente analizar con detenidamente tales desviaciones, antes de aplicar la distribución. Las causas pueden ser diversas. En todo caso, se sugiere propiciar un análisis de las mimas y ejemplificar. Por ejemplo, una causa podría asociarse a que la muestra pueda haberse elegido de una población que no es homogénea para la variable estudiada: tal es el caso de la concentración en plasma de isoniacida después de administrar una dosis estándar en grupos de personas con polimorfismo genético para la enzima metabolizante (Bowman y Raud, 1984). Otra estriba en que si bien la población es homogénea, la distribución de frecuencia de los valores es diferente. La elección de una escala de medición que no es apropiada, puede dar lugar a tales desviaciones.

Aplicaciones útiles de la distribución normal en tópicos de estadística inferencial referidos a la Medicina se pueden encontrar en otros textos complementarios para la carrera (Oliva y O'Farril, 1984), aunque susceptibles de una actualización. 
Es recomendable sugerir a los estudiantes el tratamiento más exhaustivo de tal distribución desde el punto de vista cuantitativo, cualitativo y sus diversos usos para lo cual pueden consultarse (D’Agostino, Sullivan y Beiser, 2006; Bluman, 2009; Lancaster, 2011).

\section{Conclusiones}

A partir del sistema de distribuciones continuas univariantes de Karl Pearson se dedujo la expresión matemática de la función de densidad de la distribución normal, lo cual constituye un enfoque alternativo para introducirla en la asignatura Bioestadística, método que aporta consistencia, rigor y armonía al conocimiento estadístico matemático incluido en el programa de Informática Médica II para los estudiantes de Medicina.

El método sugerido para estudiar la distribución normal en un estadio inicial del programa, teniendo en cuenta el enfoque de familias de distribuciones que satisfacen cierta ecuación diferencial, requirió la familiarización con algunos conceptos referidos al Cálculo Diferencial, las ecuaciones diferenciales ordinarias y sus métodos de solución si bien las nociones de derivación, integración y sus respectivas interpretaciones, se exponen en unidad y coherencia.

La deducción de la forma matemática de la distribución o al menos la explicación en términos generales, del procedimiento para obtener la expresión analítica de la densidad de probabilidad, contribuyó, a partir del conjunto de recomendaciones sugeridas, a la comprensión de las aplicaciones prácticas de la distribución normal en la carrera de Medicina.

\section{Bibliografía}

Ander-Egg, E. (1995). Métodos y Técnicas de investigación social. Vol. III: Cómo organizar el trabajo de investigación. Lumen: Buenos Aires. [Consultado: 26 de octubre de 2019]. Disponible en:

https://epiprimero.files.wordpress.com/2012/01/a nder-egg-tecnicas-de- investigacionsocial.pdf

Bluman, A.G. (2009). Elementary Statistics: A Step by Step Approach. McGraw-Hill Companies, Inc.: New York.

Bowman, W.C.; Raud, M.J. (1984). Farmacología. Bases bioquímicas y patológicas. Aplicaciones clínicas. Interamericana: México D.F.
Chistiakov, B.P. (1982). Curso de Teoría de las probabilidades. Moscú: Nauka (en idioma Ruso).

D’Agostino, R.B., Sullivan, L. M. and Beiser, A.S. (2006). Introductory applied biostatistics. Thomson, Brooks/Cole: Toronto.

Dawkins, P. (2007). Differential equations. [consultado: 7 de enero de 2020]. Disponible en:

https://www.google.com/search?q=Dawkins\%2C + P.+\%282007\%29.+Differential+equation s. $++\&$ ie $=$ utf $-8 \&$ oe $=$ utf $-8 \&$ client $=$ firefox b.pdf

Escalona Fernández, L.A. (2013). Resolución de problemas matemáticos aplicados a la medicina y su impacto en la formación del médico general". Correo Científico Médico Holguín; volumen: vol. 17, No. 24

Escalona Fernández, L.A., Velázquez, J.R. (2012). Resolución de problemas de optimización sin el uso de límites y derivadas. Interpretaciones médicas". En: Flores, R. (ed.). Acta Latinoamericana de Matemática Educativa 25, 365-374.

Elgoltz, L. E. (2010). Ecuaciones diferenciales y cálculo variacional. Félix Varela: La Habana.

Freund, J.E. y Perles, B.M. (2007). Modern Elementary Statistics (12th International Editions). Pearson Prentice Hall: New Jersey.

Giacosa, N., Vergara, M. L., Zang, C., López, J., Galeano, R., Godoy, N., Maidana, J. y Such, A. (2015). Libros de texto y Programas Analíticos de Física en carreras de Ingeniería de la UNaM. Revista de Enseñanza de la Física, 199-207, 27 (no extra).

Gnedenko, B.V. (1997). The theory of probability. Overseas Publishers Association: Netherlands.

Guerra Bustillo, C.W., Menéndez Acuña, E., Barrera Morera, R. y Egaña Morales, E. (1991). . Estadística. La Habana: Pueblo y Educación.

Herrerías Pleguezuelo, R. y Callejón Céspedes, J. (2017). Los sistemas de Pearson como generadores de distribuciones de probabilidad. Aplicaciones estadísticas $\mathrm{y}$ 
económicas. [consultado: 28 de junio de 2017]. Disponible en: www.ugr.es/ callejon/lossistemas.pdf

Koroliuk, V.V. (1986). Manual de la teoría de las probabilidades y estadística matemática. Moscú: Mir.

Lancaster, H.O. (2011). Quantitative Methods in Biological and Medical Sciences: A Historical Essay. Springer: New York.

Oliva González, L., O’Farril Mons, E. (1988). Bioestadística y computación: guía de estudio. La Habana: Pueblo y Educación.

Torres Delgado, J.A., et al. (2004). Informática Médica, tomo II Bioestadística. Centro de
Cibernética Aplicada a la Medicina (CECAM). Editorial Ciencias Médicas: La Habana, 2004.

Valdés Castro, C., Sánchez Fernández, C. (2011). Introducción al Análisis Matemático. La Habana: Félix Varela.

Weisstein, Eric W. (2017) Pearson System. MathWorld, A Wolfram Web Resource. [consultado: 24 de enero de 2020]. Disponible en: http://mathworld.wolfram.com/PearsonSys tem.html

Whittle, P. (1982). Probabilidad. Moscú: Nauka (en idioma Ruso). 